Canadian University Music Review

Revue de musique des universités canadiennes

\title{
The Classification of Music: A Survey Study
}

Robert Falck et Maurice Esses

Numéro 1, 1980

URI : https://id.erudit.org/iderudit/1013737ar

DOI : https://doi.org/10.7202/1013737ar

Aller au sommaire du numéro

Éditeur(s)

Canadian University Music Society / Société de musique des universités

canadiennes

ISSN

0710-0353 (imprimé)

2291-2436 (numérique)

Découvrir la revue

\section{Citer cet article}

Falck, R. \& Esses, M. (1980). The Classification of Music: A Survey Study. Canadian University Music Review / Revue de musique des universités

canadiennes, (1), 106-129. https://doi.org/10.7202/1013737ar

All Rights Reserved (C Canadian University Music Society / Société de musique des universités canadiennes, 1980
Ce document est protégé par la loi sur le droit d'auteur. L’utilisation des services d'Érudit (y compris la reproduction) est assujettie à sa politique d'utilisation que vous pouvez consulter en ligne.

https://apropos.erudit.org/fr/usagers/politique-dutilisation/ 


\title{
THE CLASSIFICATION OF MUSIC: A SURVEY STUDY
}

\author{
Robert Falck \\ Maurice Esses
}

\section{Purpose}

The terminology used in any discipline should serve as a means of expressing concepts and theories in a clear, coherent fashion. However, when the set of connotations and ambiguities surrounding a particular term is allowed to settle unnoticed into the background, unintentional confusion almost inevitably arises. For musical terminology, two tendencies may be identified. On the one hand, the use of a particular term passively reflects prevailing attitudes and values. On the other hand, a term may be used in such a way that it functions actively in an adverse manner, by perpetuating outdated concepts and restricting the range and kind of ideas expressed. This second tendency is particularly important because it is rarely perceived on a conscious level.

These concerns grew out of discussion in a seminar on musical terminology at the University of Toronto in the academic year 1976-77. In the course of our investigation of the common classification system of music with "classical" and "popular" at opposite poles, we discovered that our historical investigations were hampered by the lack of any clear understanding of how these terms are currently used and understood. This study resulted from our attempt to discover how this informal system of classification functions among undergradute students studying music. ${ }^{1}$

\section{The Questionnaire}

To this end, we devised a questionnaire which, it was hoped, would reflect the various background connotations and 
ambiguities surrounding the terms chosen for study. In order to limit the scope of the investigation, four terms were chosen for the questionnaire: classical, popular, folk, and modern. For each term, six kinds of questions were asked: (1) respondents were asked to pick words from a list which seemed to them opposite in meaning to the terms studied, and (2) words applicable to the concept; (3) names of composers, performers, and compositions were offered for choice as examples of one classification or another, and (4) brief characterizations were likewise offered for choice. In order to allow for, and, indeed, encourage, the widest possible range of interpretation, respondents were invited to check as many of the items offered for choice as they felt were appropriate. ${ }^{2}$ (5) Two questions attempted to determine the respondent's self-assessed level of knowledge of classical music, and to compare this with the knowledge of the "average person," and (6) two final questions asked respondents to rank the four kinds of music in order of their importance for "contemporary cultural life" and "to you personally." Responses to these questions help to put the data obtained into perspective by indirectly indicating individual biases, and by identifying the self-assessed place of the respondents in the socio-cultural hierarchy. The questionnaire is reproduced below:

Please do not identify yourself on this questionnaire. This is not a test of your knowledge, but an investigation of the current understanding of a few terms as applied to music.

1. Which of the following words are opposite in meaning to the word "classical"? (Check as many as apply)

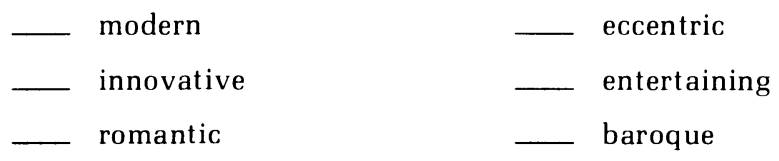

2. Which of the following adjectives are applicable to the concept of classical music? (Check as many as apply)

$\begin{array}{ll}\text { intellectual } & - \text { enduring } \\ \text { accessible } & - \text { entertaining } \\ - \text { inaccessible } & - \text { elite }\end{array}$

3. Which of the following are "classical"? (Check as many as apply)

$\begin{array}{ll}\text { Machaut } & - \text { Healey Willan } \\ - \text { Palestrina } & - \text { Chaikovsky } \\ - \text { Boston Pops } & - \text { Chopin }\end{array}$


_ _ Bartók

- Ravi Shankar

- Mozart

4. How much classical music do you think the average person is familiar with? (Check one)

- Almost none

- A little

- A lot

5. How much classical music are you familiar with? (Check one)

_ Almost none

- A little

- A lot

6. Which of the following describe the meaning of the word "classical" in music? (Check as many as apply)

A musical style

- A historical time period

- An aeshtetic attitude

- An ideal standard

- An established norm

- A value judgement

7. Which of the following words are opposite in meaning to the word "popular"? (Check as many as apply)

$\begin{array}{ll}\text { serious } & - \text { elite } \\ - \text { accessible } & - \text { conservative } \\ - \text { inaccessible } & - \text { expensive }\end{array}$

8. Which of the following words are applicable to the concept of popular music? (Check as many as apply)

$\begin{array}{ll}\text { serious } & - \text { cheap } \\ \text { common } & - \text { entertaining } \\ - \text { relevant } & - \text { traditional }\end{array}$

9. Which of the following would you classify as "popular"? (Check as many as apply)

$\begin{array}{ll}\text { - Petula Clark } & - \text { B.T.O. } \\ - \text { Louis Armstrong } & - \text { Joan Baez } \\ -\begin{array}{l}\text { Boston Pops } \\ - \text { "West Side Story" }\end{array} & -\quad \text { "The Mikado" } \\ - & - \text { Mozart }\end{array}$


10. Which of the following describe the meaning of the word "popular"? (Check as many as apply)

- A musical style

A contemporary phenomenon

A social standing

A value judgement

An ideal standard

11. Which of the following words are opposite in meaning to the word "folk"? (Check as many as apply)

$\begin{array}{ll}- \text { serious } & - \text { conservative } \\ - \text { vulgar } & - \text { popular } \\ - \text { sophisticated } & - \text { elite }\end{array}$

12. Which of the following words are applicable to the concept of folk music? (Check as many as apply)

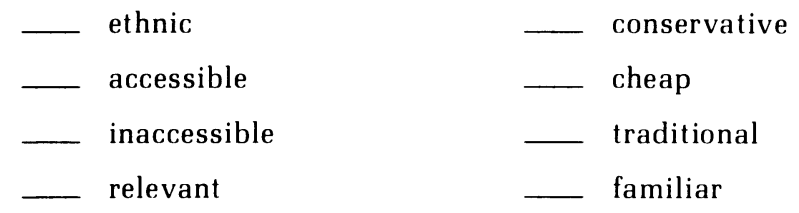

13. Which of the following would you classify as "folk"? (Check as many as apply)

$\begin{array}{ll}\text { - Gordon Lightfoot } & - \text { The Beatles } \\ - \text { Bob Dylan } & - \text { Joan Baez } \\ - \text { "Porgy and Bess" } & - \text { blues } \\ - \text { Bartók } & - \text { Ravi Shankar }\end{array}$

14. Which of the following describe the meaning of the word "folk" in music? (Check as many as apply)
- A musical style
- An ethnic classification
- An established norm
- A contemporary phenomenon
A value judgement

15. Which of the following words are opposite in meaning to the word "modern"? (Check as many as apply)
_ ancient
__ conservative
_ classical
__ contemporary
_ romantic
- radical 
16. Which of the following adjectives are applicable to the concept of modern music? (Check as many as apply)

$\begin{array}{ll}\text { progressive } & - \text { classical } \\ - \text { accessible } & - \text { popular } \\ - \text { inaccessible } & - \text { radical }\end{array}$

17. Which of the following would you classify as "modern"? (Check as many as apply)

$\begin{array}{ll}\text { - Stockhausen } & - \text { Healy Willan } \\ - \text { Bob Dylan } & - \text { Wagner } \\ - \text { "Jesus Christ, Superstar" } & - \text { Gershwin } \\ - \text { Bartók } & - \text { Oscar Peterson }\end{array}$

18. Which of the following describe the meaning of the word "modern" in music? (Check as many as apply)
_ A musical style
An aesthetic attitude
- An historical time period
A contemporary phenomenon
_ An ideal standard
A value judgement

19. Rank the four kinds of music in order of their importance for contemporary cultural life. $(1=$ most important $)$
- classical
_ modern
- popular
folk

20. Rank the four kinds of music in order of their value to you personally. ( 1 = most valuable)
- classical
modern
- popular
folk

Figure I

The first two question types ("opposite," nos. 1, 7, 11, 15; "applicable," nos, $2,8,12,16$ ) would appear to be redundant, as they draw on a common pool of adjectives, and elicit basically the same kind of information. However, by placing the "opposite" questions first, respondents. were perhaps induced to select answers more spontaneously than if more direct ("applicable") questions were given precedence. Furthermore, inconsistencies which can occur provide grounds for considering particular responses invalid, especially in cases where the same word appears under each question type for a given term. For 
example, "entertaining" appears in questions 1 \& 2, "serious" in $7 \& 8$, and "conservative" in 11 \& 12 .

\section{Composition of the Sample Group}

The group of respondents to our questionnaire (the "sample") consisted of 261 undergraduate students at the University of Toronto. These students ranged in age and musical experience from first year general Arts students with no previous musical training to fourth year students preparing to enter graduate study in musicology. ${ }^{3}$ In the statistical tables which follow, they are divided into three large groups as follows:

I. Arts and Science Students

A. MUS 100 (50 questionnaires). This is an introductory course in Western music history and literature for non-music students.

B. MUS 200 (17 questionnaires). Parallel to the above, but covering non-Western music.

II. Faculty of Music, First and Second years

A. HMU 120 (82 questionnaires). Introduction to music history for music majors, covering all historical periods. Compulsory for Faculty of Music students.

B. HMU 220 (53 questionnaires). Continuation of the above; a more detailed survey from Haydn to the present.

III. Faculty of Music, Third and Fourth years

A. HMU 454 - "Beethoven" (29 questionnaires). An elective course for upper level undergraduates, predominantly History and Literature majors.

B. HMU 354 - "Concepts of Popular Music" (13 questionnaires). As above, covering popular music of all periods.

C. HMU 357 - "Impressionism and Expressionism" (17 questionnaires). As above, covering early twentieth-century music in Germany and France.

The character of the various sub-groups has been set out in some detail because, contrary to expectations, the subject matter of the course in which the students were engaged did 
have some influence on their responses. Except for students in HMU 120 and 220, all were in "elective" courses, so it is unclear whether their experience in the course influenced their answers, or whether their previous experience influenced them to take the course.

\section{Overall Quantitative Results}

The complete results of the surveys are set out below. Figures given represent the percentage of respondents choosing each individual item on the questionnaire, and sub-totals are shown for all Arts students (column 3), all first and second year Faculty of Music students (column 6), all third and fourth year Faculty of Music students (column 10), all Faculty of Music students (column 11), and all respondents (column 12). Additional data concerning the combination of rankings in questions 19 and 20 taken together within each questionnaire is particularly important. In the interests of simplicity we have avoided presenting all $576(24 \times 24)$ such possible combinations. Instead, the results for the pairs of terms ranked first in both questions are provided in Table 1a.

\section{Biases of the Sample Group (especially questions 4-5; 19-20)}

Some of the extreme deviations in Table I reveal differences among the groups based upon background, experience, and subject matter. Without examining the statistics in detail, several such instances may be noted: (1) In question 3, Ravi Shankar was chosen as an example of "classical" by $35 \%$ of MUS 200 students, and $31 \%$ of HMU 364 students, against an overall average of $12 \%$. Awareness of Shankar and of his status as a classical musician was evidently more highly developed in those students taking subjects outside the main stream of Western "classical" music. (2) The same explanation may be offered for HMU 364's markedly different reaction to the adjective "serious" in relation to "popular." Only 15\% chose it as opposite to "popular" (overall 41\%), and 31\% chose it as applicable (overall 15\%). (3) In question 9, HMU 364 again proved to be the most clearly deviant group. Both "West-Side Story" and "Louis Armstrong" were chosen by $85 \%$ as representative of "popular" (42\% and $43 \%$ respectively overall), and $69 \%$ chose "The Mikado" (overall 23\%). Again, the different perspective of these students had evidently altered and broadened their 
concept of "popular." (4) HMU 364 picked "blues" as representative of "folk" in question $1346 \%$ of the time, against an average overall of $23 \%$. (5) In question 17, Karlheinz Stockhausen was identified as "modern" by $100 \%$ of HMU $357,92 \%$ of HMU 364 , and $89 \%$ of HMU 220 . This compares to an overall response of $65 \%$, and a $40 \%$ response among Arts and Science students. This suggests not so much a difference of opinion as a difference in knowledge and experience. The groups which chose Stockhausen less frequently may have done so because he was unknown to them. This is suggested most strongly by the varied response of HMU 120 and 220, as students in 220 had just finished studying twentieth-century music when they completed the questionnaire, whereas the HMU 120 group had not yet "covered" that repertory.

These differences between and among groups focus largely on their varied responses to "classical" and "non-classical" categories. Questions 4-5, 19-20 were aimed at discovering the group's own estimate of their biases toward the four categories studied, and their assessment of the "average person's" knowledge. The statistical tables tell only part of this story, as only percentages for individual categories are shown. The combinations chosen in questions $4-5$ and in 19 and 20 are needed to complete the picture.

Combining the responses to questions 4 and 5 , one finds that no individual considered that he or she was familiar with less "classical music" than the average person. Only $22 \%$ of the respondents considered their knowledge of "classical music" on a par with that of the average person. The largest group $-67 \%$ - ranked their knowledge one degree higher than that of the "average person." That is, they chose one of the following combinations: 4. Almost none \& 5 . A little; or 4. A little \& 5 . A lot. The remaining $10 \%$ chose the most contrasting combination of "almost none" in question 4, and "a lot" in question 5. 
Question 1 modern innovative romantic eccentric entertaining baroque

Question 2 intellectual accessible inaccessible enduring entertaining elite

Question 3

Machaut

Palestrina

Boston Pops

Bartók

Willan

Chaikovsky

Chopin

Shankar

Mozart

Question 4

Almost none

A little

A lot

Question 5

Almost none

A little

A lot

Question 6 musical style time period aesthetic standard norm value

\begin{tabular}{|c|c|c|c|c|c|c|c|c|c|c|c|}
\hline 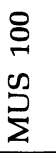 & $\begin{array}{l}\text { 尺े } \\
\text { D } \\
己 \\
己\end{array}$ & $\begin{array}{l}-\infty \\
\mathbb{0} \infty \\
0 \\
0\end{array}$ & 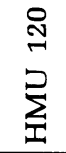 & $\begin{array}{l}\text { సิ } \\
\text { ᄅ } \\
\sum_{\text {I }}\end{array}$ & $\underset{0}{\pi}$ & 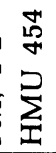 & $\begin{array}{l}\stackrel{D}{0} \\
\sum^{2} \\
己\end{array}$ & 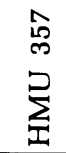 & $\underset{\oplus}{\stackrel{\pi}{\sigma}}$ & $\stackrel{\pi}{0} \sum_{[x}$ & $\stackrel{\pi}{0}$ \\
\hline 70 & 47 & 43 & 59 & 63 & 60 & 52 & 38 & 47 & 47 & 56 & 58 \\
\hline 20 & 6 & 16 & 26 & 30 & 27 & 3 & 8 & 12 & 7 & 21 & 20 \\
\hline 30 & 35 & 31 & 34 & 53 & 41 & 55 & 31 & 41 & 46 & 43 & 40 \\
\hline 30 & 12 & 25 & 30 & 34 & 32 & 21 & 23 & 12 & 19 & 28 & 26 \\
\hline 6 & 12 & 7 & 5 & 13 & 8 & 7 & 8 & 6 & 7 & 8 & 8 \\
\hline 24 & 53 & 31 & 32 & 43 & 36 & 48 & 38 & 18 & 37 & 37 & 35 \\
\hline 72 & 71 & 66 & 71 & 66 & 69 & 69 & 54 & 59 & 63 & 67 & 68 \\
\hline 38 & 47 & 40 & 45 & 40 & 43 & 45 & 46 & 47 & 46 & 44 & 43 \\
\hline 8 & 0 & 6 & 5 & 4 & 4 & 0 & 15 & 0 & 3 & 4 & 5 \\
\hline 50 & 47 & 49 & 56 & 58 & 57 & 52 & 54 & 82 & 61 & 71 & 66 \\
\hline 40 & 35 & 39 & 39 & 49 & 43 & 55 & 38 & 47 & 49 & 45 & 43 \\
\hline 30 & 53 & 36 & 40 & 28 & 36 & 24 & 46 & 35 & 32 & 35 & 35 \\
\hline 23 & 18 & 18 & 21 & 9 & 16 & 10 & 23 & 35 & 20 & 18 & 18 \\
\hline 14 & 41 & 21 & 24 & 13 & 20 & 14 & 23 & 47 & 25 & 22 & 21 \\
\hline 6 & 6 & 6 & 11 & 2 & 7 & 0 & 15 & 12 & 10 & 7 & 7 \\
\hline 22 & 41 & 27 & 26 & 8 & 18 & 14 & 31 & 35 & 24 & 20 & 22 \\
\hline 4 & 12 & 6 & 23 & 6 & 16 & 3 & 31 & 18 & 14 & 15 & 13 \\
\hline 38 & 59 & 43 & 39 & 21 & 32 & 17 & 31 & 24 & 22 & 24 & 29 \\
\hline 36 & 59 & 42 & 35 & 15 & 27 & 21 & 31 & 29 & 25 & 27 & 31 \\
\hline 6 & 35 & 13 & 10 & 8 & 9 & 7 & 31 & 29 & 19 & 12 & 12 \\
\hline 94 & 94 & 94 & 100 & 100 & 100 & 93 & 100 & 100 & 97 & 99 & 98 \\
\hline 34 & 47 & 37 & 39 & 28 & 35 & 24 & 15 & 24 & 22 & 36 & 36 \\
\hline 66 & 53 & 63 & 59 & 64 & 61 & 76 & 85 & 76 & 78 & 66 & 65 \\
\hline 0 & 0 & 0 & 2 & 2 & 2 & 0 & 0 & 0 & 0 & 1 & 1 \\
\hline 0 & 6 & 1 & 0 & 0 & 0 & 0 & 0 & 0 & 0 & 0 & .4 \\
\hline 62 & 59 & 61 & 45 & 24 & 37 & 41 & 15 & 23 & 30 & 35 & 42 \\
\hline 38 & 35 & 37 & 55 & 76 & 63 & 59 & 85 & 76 & 69 & 65 & 58 \\
\hline 76 & 76 & 76 & 89 & 92 & 90 & 86 & 92 & 76 & 85 & 89 & 85 \\
\hline 70 & 53 & 66 & 77 & 81 & 79 & 76 & 77 & 65 & 73 & 77 & 74 \\
\hline 40 & 47 & 42 & 43 & 49 & 45 & 59 & 62 & 65 & 61 & 50 & 47 \\
\hline 20 & 12 & 18 & 21 & 17 & 19 & 10 & 31 & 12 & 15 & 18 & 18 \\
\hline 10 & 12 & 10 & 21 & 17 & 19 & 10 & 23 & 18 & 15 & 18 & 16 \\
\hline 4 & 29 & 10 & 15 & 8 & 12 & 7 & 31 & 47 & 24 & 15 & 14 \\
\hline
\end{tabular}


Question 7

serious

accessible

inaccessible

elite

conservative

expensive

Question 8

serious

common

relevant

cheap

entertaining

traditional

Question 9

Petula Clark

Louis Armstrong

Boston Pops

West Side Story

B.T.O.

Joan Baez

"Mikado"

Mozart

Question 10

musical style

social standing

ideal standard

contemporary

value

Question 11

serious

vulgar

sophisticated

conservative

popular

elite

Question 12

ethnic

accessible

inaccessible

relevant

conservative

cheap

traditional

familiar

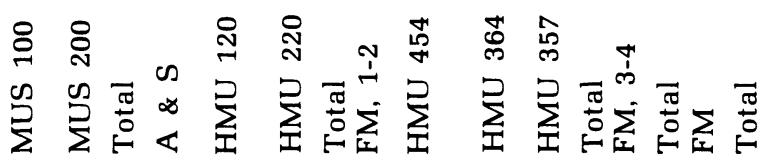

\begin{tabular}{|c|c|c|c|c|c|c|c|c|c|c|c|}
\hline 50 & 29 & 45 & 39 & 60 & 47 & 24 & 15 & 29 & 24 & 40 & 41 \\
\hline 14 & 0 & 10 & 5 & 9 & 7 & 10 & 15 & 12 & 12 & 8 & 9 \\
\hline 40 & 65 & 46 & 49 & 42 & 46 & 41 & 54 & 59 & 49 & 47 & 47 \\
\hline 42 & 65 & 48 & 57 & 39 & 50 & 52 & 46 & 76 & 58 & 53 & 51 \\
\hline 34 & 24 & 31 & 30 & 28 & 30 & 17 & 8 & 0 & 9 & 23 & 26 \\
\hline 6 & 18 & 9 & 12 & 11 & 12 & 3 & 8 & 8 & 5 & 10 & 10 \\
\hline 12 & 18 & 13 & 18 & 9 & 15 & 21 & 31 & 6 & 19 & 16 & 15 \\
\hline 80 & 59 & 75 & 72 & 62 & 68 & 69 & 62 & 71 & 68 & 68 & 70 \\
\hline 28 & 53 & 34 & 44 & 36 & 41 & 24 & 77 & 35 & 39 & 40 & 39 \\
\hline 34 & 12 & 28 & 24 & 28 & 26 & 14 & 15 & 6 & 12 & 24 & 25 \\
\hline 88 & 88 & 88 & 61 & 87 & 71 & 79 & 85 & 82 & 81 & 74 & 78 \\
\hline 10 & 12 & 10 & 16 & 17 & 16 & 7 & 38 & 6 & 14 & 15 & 14 \\
\hline 74 & 76 & 75 & 68 & 72 & 70 & 83 & 85 & 88 & 85 & 74 & 74 \\
\hline 42 & 65 & 48 & 44 & 28 & 38 & 27 & 85 & 53 & 47 & 41 & 43 \\
\hline 54 & 65 & 57 & 70 & 43 & 59 & 52 & 77 & 65 & 61 & 60 & 59 \\
\hline 46 & 65 & 51 & 49 & 34 & 43 & 31 & 85 & 65 & 53 & 46 & 47 \\
\hline 58 & 65 & 60 & 83 & 58 & 73 & 52 & 69 & 76 & 63 & 70 & 67 \\
\hline 54 & 76 & 60 & 62 & 55 & 59 & 59 & 77 & 59 & 63 & 60 & 60 \\
\hline 10 & 12 & 10 & 22 & 24 & 23 & 17 & 69 & 53 & 39 & 28 & 23 \\
\hline 2 & 6 & 3 & 13 & 9 & 12 & 14 & 38 & 24 & 22 & 15 & 12 \\
\hline 52 & 41 & 49 & 44 & 45 & 44 & 48 & 46 & 29 & 42 & 44 & 45 \\
\hline 26 & 47 & 31 & 40 & 38 & 39 & 17 & 31 & 24 & 22 & 34 & 33 \\
\hline 2 & 6 & 3 & 10 & 2 & 7 & 3 & 0 & 6 & 3 & 6 & 5 \\
\hline 72 & 71 & 72 & 62 & 57 & 60 & 62 & 38 & 41 & 51 & 57 & 61 \\
\hline 30 & 47 & 34 & 43 & 30 & 38 & 21 & 54 & 47 & 36 & 37 & 36 \\
\hline 14 & 23 & 16 & 26 & 15 & 21 & 14 & 15 & 6 & 12 & 19 & 18 \\
\hline 8 & 18 & 10 & 24 & 19 & 22 & 10 & 8 & 6 & 8 & 18 & 16 \\
\hline 58 & 53 & 57 & 58 & 55 & 57 & 62 & 54 & 53 & 58 & 57 & 57 \\
\hline 18 & 29 & 21 & 26 & 9 & 19 & 17 & 8 & 0 & 10 & 16 & 18 \\
\hline 16 & 6 & 13 & 7 & 4 & 6 & 7 & 8 & 0 & 5 & 6 & 8 \\
\hline 46 & 65 & 51 & 55 & 51 & 53 & 62 & 46 & 71 & 61 & 55 & 54 \\
\hline 80 & 88 & 82 & 79 & 87 & 82 & 29 & 77 & 100 & 81 & 82 & 82 \\
\hline 50 & 82 & 58 & 61 & 51 & 57 & 62 & 69 & 71 & 66 & 60 & 59 \\
\hline 0 & 0 & 0 & 2 & 2 & 2 & 3 & 0 & 0 & 2 & 2 & 2 \\
\hline 46 & 47 & 46 & 34 & 42 & 37 & 45 & 54 & 47 & 47 & 40 & 42 \\
\hline 12 & 6 & 10 & 11 & 19 & 14 & 10 & 23 & 18 & 15 & 14 & 13 \\
\hline 4 & 12 & 6 & 7 & 8 & 7 & 7 & 8 & 0 & 5 & 7 & 6 \\
\hline 94 & 53 & 84 & 78 & 79 & 78 & 59 & 92 & 88 & 75 & 77 & 79 \\
\hline 72 & 47 & 66 & 65 & 70 & 67 & 76 & 54 & 71 & 69 & 67 & 67 \\
\hline
\end{tabular}


Question 13

Lightfoot

Dylan

"Porgy \& Bess"

Bartók

Beatles

Joan Baez

blues

Shankar

Question 14

musical style

ethnic

established norm

contemporary

value

Question 15

ancient

classical

romantic

conservative

contemporary

radical

Question 16

progressive

accessible

inaccessible

classical

popular

radical

Question 17

Stockhausen

Dylan

J.C., Superstar

Bartók

Willan

Wagner

Gershwin

Peterson

Question 18

musical style

period

ideal

aesthetic

contemporary

value

\begin{tabular}{|c|c|c|c|c|c|c|c|c|c|c|c|}
\hline 용 & 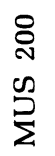 & $\begin{array}{l}\pi^{\infty} \\
0_{0}^{\infty} \\
6\end{array}$ & 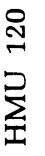 & $\begin{array}{l}\text { 今 } \\
\text { N } \\
己 \\
己\end{array}$ & $\frac{N}{\stackrel{N}{*}}$ & $\begin{array}{l}\text { 畒 } \\
\sum^{2}\end{array}$ & 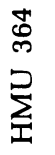 & $\begin{array}{l}\stackrel{\hat{\omega}}{\infty} \\
\sum_{1}^{2} \frac{\pi}{0}\end{array}$ & $\sum_{i=}^{+}$ & $\stackrel{\pi}{0} \sum_{i}$ & 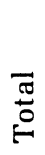 \\
\hline 64 & 71 & 66 & 88 & 81 & 85 & 83 & 46 & 53 & 66 & 79 & 76 \\
\hline 60 & 71 & 63 & 84 & 72 & 79 & 76 & 69 & 65 & 71 & 77 & 73 \\
\hline 18 & 12 & 16 & 8 & 11 & 10 & 21 & 31 & 41 & 28 & 15 & 16 \\
\hline 14 & 6 & 12 & 12 & 30 & 19 & 21 & 54 & 41 & 34 & 24 & 20 \\
\hline 12 & 6 & 10 & 6 & 4 & 5 & 0 & 8 & 0 & 2 & 4 & 6 \\
\hline 70 & 76 & 72 & 79 & 72 & 76 & 83 & 69 & 65 & 75 & 76 & 75 \\
\hline 28 & 35 & 30 & 13 & 13 & 13 & 31 & 46 & 35 & 36 & 20 & 23 \\
\hline 14 & 18 & 15 & 13 & 13 & 13 & 14 & 15 & 12 & 14 & 13 & 14 \\
\hline 76 & 53 & 70 & 78 & 68 & 74 & 76 & 54 & 41 & 61 & 70 & 70 \\
\hline 62 & 71 & 64 & 57 & 68 & 61 & 65 & 77 & 76 & 71 & 64 & 64 \\
\hline 10 & 35 & 16 & 18 & 19 & 18 & 17 & 8 & 18 & 15 & 17 & 17 \\
\hline 8 & 6 & 7 & 16 & 9 & 13 & 34 & 15 & 6 & 22 & 16 & 14 \\
\hline 20 & 29 & 22 & 19 & 9 & 16 & 17 & 31 & 29 & 24 & 18 & 19 \\
\hline 80 & 88 & 82 & 85 & 83 & 84 & 86 & 77 & 94 & 86 & 85 & 84 \\
\hline 72 & 53 & 67 & 59 & 51 & 56 & 69 & 31 & 35 & 59 & 57 & 59 \\
\hline 40 & 24 & 37 & 43 & 45 & 44 & 59 & 23 & 12 & 37 & 42 & 40 \\
\hline 36 & 24 & 33 & 33 & 42 & 36 & 24 & 15 & 12 & 19 & 31 & 31 \\
\hline 2 & 0 & 1 & 4 & 4 & 4 & 0 & 0 & 6 & 2 & 3 & 3 \\
\hline 8 & 0 & 6 & 0 & 2 & 1 & 0 & 0 & 0 & 0 & .5 & 2 \\
\hline 70 & 65 & 69 & 80 & 75 & 78 & 69 & 77 & 65 & 69 & 76 & 74 \\
\hline 26 & 53 & 33 & 34 & 19 & 28 & 28 & 31 & 6 & 22 & 26 & 28 \\
\hline 22 & 6 & 18 & 16 & 30 & 21 & 10 & 0 & 24 & 12 & 19 & 15 \\
\hline 5 & 12 & 4 & 21 & 8 & 15 & 0 & 23 & 18 & 10 & 14 & 11 \\
\hline 36 & 35 & 36 & 26 & 11 & 20 & 21 & 31 & 12 & 20 & 20 & 24 \\
\hline 56 & 29 & 49 & 56 & 74 & 63 & 52 & 54 & 24 & 44 & 57 & 55 \\
\hline 38 & 47 & 40 & 59 & 89 & 70 & 69 & 92 & 100 & 83 & 74 & 65 \\
\hline 32 & 41 & 49 & 34 & 25 & 30 & 24 & 62 & 59 & 42 & 34 & 38 \\
\hline 58 & 59 & 58 & 43 & 32 & 38 & 38 & 62 & 53 & 47 & 41 & 46 \\
\hline 32 & 35 & 33 & 55 & 43 & 50 & 45 & 46 & 41 & 44 & 48 & 48 \\
\hline 32 & 24 & 30 & 40 & 28 & 35 & 24 & 46 & 24 & 29 & 33 & 33 \\
\hline 2 & 0 & 1 & 12 & 13 & 13 & 3 & 15 & 18 & 10 & 12 & 9 \\
\hline 40 & 35 & 39 & 43 & 23 & 35 & 34 & 69 & 29 & 41 & 37 & 37 \\
\hline 38 & 47 & 40 & 44 & 21 & 35 & 38 & 54 & 47 & 41 & 37 & 37 \\
\hline 64 & 35 & 57 & 55 & 64 & 58 & 52 & 38 & 29 & 42 & 53 & 54 \\
\hline 52 & 47 & 51 & 50 & 55 & 52 & 59 & 54 & 41 & 53 & 48 & 49 \\
\hline 6 & 0 & 4 & 9 & 8 & 8 & 10 & 8 & 6 & 8 & 8 & 7 \\
\hline 30 & 29 & 30 & 44 & 43 & 44 & 31 & 31 & 47 & 36 & 41 & 38 \\
\hline 34 & 53 & 39 & 50 & 49 & 50 & 45 & 46 & 35 & 42 & 47 & 45 \\
\hline 18 & 18 & 18 & 24 & 19 & 22 & 10 & 23 & 41 & 22 & 22 & 21 \\
\hline
\end{tabular}


Question 19 classical 1 2
3 folk 1

2
3
4

modern 1 $\begin{array}{cc} & 2 \\ & 3 \\ \text { popular } & 4 \\ & 1 \\ & 2 \\ & 3 \\ & 4\end{array}$

Question 20 classical 1 2
3
4 folk 1 2
3
4 modern 1 $\begin{array}{cc} & 2 \\ & 3 \\ & 4 \\ \text { popular } & 1 \\ & 2 \\ & 3 \\ & 4\end{array}$

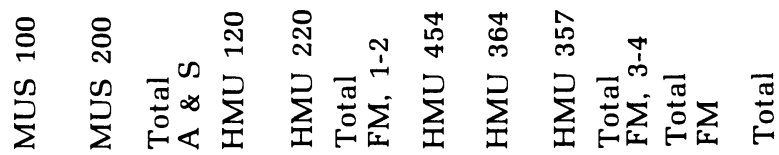

\begin{tabular}{|c|c|c|c|c|c|c|c|c|c|c|c|}
\hline 22 & 0 & 16 & 22 & 23 & 22 & 31 & 15 & 24 & 25 & 23 & 21 \\
\hline 12 & 29 & 16 & 24 & 17 & 21 & 24 & 15 & 24 & 22 & 22 & 20 \\
\hline 24 & 18 & 22 & 37 & 32 & 35 & 31 & 38 & 29 & 32 & 34 & 31 \\
\hline-22 & $3 \underline{5}$ & -25 & 13 & 15 & $-1 \underline{4}$ & 10 & $\underline{15}$ & $\underline{0}$ & $-\underline{9}$ & 12 & 16 \\
\hline$\overline{10}$ & 12 & 10 & 7 & $\overline{17}$ & 11 & $\overline{10}$ & 23 & $\overline{6}$ & 12 & 11 & 11 \\
\hline 26 & 12 & 22 & 39 & 41 & 40 & 38 & 23 & 29 & 32 & 38 & 34 \\
\hline 36 & 53 & 40 & 32 & 19 & 27 & 41 & 23 & 29 & 34 & 29 & 32 \\
\hline$\underline{8}$ & 6 & 7 & 20 & 9 & 16 & 7 & 15 & 12 & 10 & 14 & 12 \\
\hline $1 \overline{16}$ & 12 & 15 & 16 & 11 & 14 & $\overline{10}$ & 8 & 6 & 8 & 12 & $\overline{13}$ \\
\hline 24 & 35 & 27 & 21 & 9 & 16 & 14 & 31 & 24 & 20 & 18 & 20 \\
\hline 14 & 6 & 12 & $17^{\circ}$ & 19 & 18 & 10 & 8 & 6 & 8 & 15 & 14 \\
\hline 26 & 29 & 27 & 43 & 47 & 44 & $\underline{62}$ & 38 & $\underline{41}$ & $\underline{5} 1$ & 46 & 41 \\
\hline$\overline{3} \overline{2}$ & $\overline{59}$ & 39 & $\overline{48}$ & 40 & 44 & $\overline{41}$ & 38 & 41 & 41 & 43 & 42 \\
\hline 18 & 6 & 15 & 13 & 19 & 16 & 21 & 15 & 0 & 14 & 15 & 15 \\
\hline 6 & 6 & 6 & 11 & 11 & 11 & 17 & 15 & 12 & 15 & 12 & 11 \\
\hline 24 & 12 & 21 & 21 & 17 & 19 & 17 & 15 & 24 & 19 & 19 & 20 \\
\hline 48 & 35 & 45 & 67 & 72 & 69 & 83 & 38 & 65 & 68 & 69 & 62 \\
\hline 22 & 35 & 25 & 22 & 9 & 17 & 10 & 15 & 0 & 8 & 14 & 17 \\
\hline 14 & 12 & 13 & 5 & 6 & 5 & 3 & 8 & 6 & 5 & 5 & 7 \\
\hline 4 & 6 & 4 & 1 & $\underline{0}$ & 1 & 0 & 8 & 0 & 2 & 1 & $\underline{2}$ \\
\hline 14 & $\overline{18}$ & $\overline{1} 5$ & $\overline{1}$ & $\overline{2}$ & $\overline{1}$ & 7 & $\overline{8}$ & 0 & 5 & 3 & 6 \\
\hline 28 & 29 & 28 & 17 & 34 & 24 & 52 & 25 & 29 & 37 & 28 & 28 \\
\hline 26 & 35 & 28 & 41 & 41 & 41 & 17 & 23 & 29 & 22 & 36 & 34 \\
\hline 20 & 6 & 16 & 34 & 9 & 24 & 21 & 23 & 12 & 19 & 23 & 21 \\
\hline 18 & 24 & $\overline{19}$ & 15 & $\overline{8}$ & 12 & $\overline{0}$ & 0 & 6 & 2 & 9 & 11 \\
\hline 14 & 12 & 13 & 35 & 28 & 33 & 17 & 15 & 24 & 19 & 28 & 25 \\
\hline 26 & 24 & 25 & 20 & 17 & 18 & 28 & 15 & 24 & 24 & 20 & 21 \\
\hline-30 & 29 & 30 & 20 & 34 & 25 & 52 & 38 & 18 & 39 & 29 & 29 \\
\hline 10 & 18 & $1 \overline{12}$ & 12 & $\overline{6}$ & $\overline{10}$ & $\overline{10}$ & 23 & $\overline{0}$ & $\overline{10}$ & 10 & 10 \\
\hline 24 & 6 & 19 & 16 & 15 & 16 & 14 & 23 & 18 & 17 & 16 & 17 \\
\hline 20 & 24 & 21 & 22 & 23 & 22 & 48 & 23 & 12 & 32 & 25 & 24 \\
\hline 34 & 41 & 36 & 39 & 43 & 41 & 24 & 0 & 41 & 24 & 36 & 36 \\
\hline
\end{tabular}

Table I

Statistical Table for all Questions (given in percentages)

The overall contrast between the survey group and the "average person" is probably even greater than these percentages show, because of the tendency in surveys to select a middle answer from a list of graduated possibilities, regardless of the question. ${ }^{4}$ 


\begin{tabular}{|c|c|c|c|c|}
\hline & \multicolumn{4}{|c|}{ First choice in Question 20} \\
\hline & classical & folk & modern & popular \\
\hline classical & 20 & 2 & 0 & 2 \\
\hline folk & 8 & 2 & 2 & 1 \\
\hline modern & 7 & 1 & 6 & .4 \\
\hline popular & 35 & 1 & 4 & 9 \\
\hline
\end{tabular}

Table Ia

Combination within each questionnaire of first choices in Questions 19 and 20 given in percentages $(100 \%=230$ questionnaires $)$

Question 5

\begin{tabular}{|c|c|c|c|}
\hline & Almost none & A little & A lot \\
\hline Almost none & .4 & 22 & 10 \\
\hline A little & 0 & 21 & 45 \\
\hline A lot & 0 & 0 & 1 \\
\hline
\end{tabular}

Table II

Combination of responses to Questions $4 \& 5$ given in percentages $(100 \%=258$ questionnaires; total $=99 \%$ because of rounding-off procedures $)$

A group bias toward "classical music," regardless of the particular meanings selected earlier by the individual respondent, is revealed by the combination answers to questions 19 and 20 . Only 6\% of the respondents ranked "classical music" lower in value to them personally (question 20) than to "contemporary cultural life" (question 19); $33 \%$ ranked it as equal in both questions; the majority - 62\% - ranked it higher in question 20 .

Ranking in Question 20

\begin{tabular}{ll|rrrc}
\multicolumn{1}{c}{} & \multicolumn{1}{c}{1} & 2 & 3 & 4 \\
\cline { 3 - 6 } Ranking in & 1 & 20 & 3 & .4 & 0 \\
Question 19 & 2 & 14 & 8 & 1 & .4 \\
& 3 & 27 & 5 & 4 & 1 \\
& 4 & 9 & 4 & 3 & 1
\end{tabular}

Table III

Combinations of ranking of "classical music" in Questions $19 \& 20$ given in percentages $(100 \%=227$ questionnaires; total $=101 \%$ because of rounding-off procedures) 
The contrast between the survey group and the generality may also be shown by examining the ranking in question 20 of the first choice in question 19. The most significant statistic would seem to be that $32 \%$ ranked "popular music" first in question 19, but third or fourth in question 20 . The other larger group is made up of the $20 \%$ who ranked "classical music" first in both questions. ${ }^{5}$

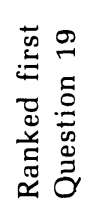

$$
\begin{array}{r}
\text { classical } \\
\text { folk } \\
\text { modern } \\
\text { popular }
\end{array}
$$

20

1
20
6
9

\begin{tabular}{lcr}
2 & 3 & \multicolumn{1}{c}{4} \\
3 & .4 & 0 \\
5 & 4 & 1 \\
5 & 3 & 1 \\
9 & 16 & 16
\end{tabular}

Table IV

Ranking in Question 20 of first choice in Question 19 given in percentages $(100 \%=225$ questionnaires $)$

The ranking of "classical music" in question 20 is likewise related to the individual's assessment of his/her knowledge of "classical music" in questions 4-5. This is shown by comparing the composition of the sub-group which ranked "classical music" first in question 20, with the sub-group ranking it second to fourth. The percentage of respondents who considered their familiarity as only roughly equal to that of the "average person" is significantly higher in the latter sub-group.

\section{Questions 4-5}

\begin{tabular}{lc|cc|c} 
Ranking in & 1 & 58 & 12 & $\begin{array}{c}\text { more } \\
\text { Question 20 }\end{array}$ \\
\hline & 2 & 12 & 7 & equal in sub-group 1 \\
& 3 & 6 & 3 & $33\left(=\frac{12}{70} \times 100\right)$ \\
& 4 & 2 & .4 & \\
\hline
\end{tabular}

Table V

Ranking of "classical" in Question 20

and self-assessed knowledge in Questions 4-5 given in percentages $(100 \%=237$ questionaires $)$ 
Although the foregoing argues for a strong commitment by the whole group surveyed toward "classical music," differences appear again if we regard the sample as made up of two or three separate groups. As shown in Table VI, there appears to be a direct relationship between the amount of training in music and the commitment to "classical music."

$\begin{array}{cc}\text { Group } & \text { Percentage } \\ \text { MUS 100 } & 42 \\ \text { MUS 200 } & 53 \\ \text { Sub-total } & 47.5 \\ \text { HMU 120 } & 29 \\ \text { HMU 220 } & 28 \\ \text { Sub-total } & 29 \\ \text { HMU 454 } & 14 \\ \text { HMU 364 } & 30 \\ \text { HMU 357 } & 12 \\ \text { Sub-total } & 19\end{array}$

Table VI

Percentage of respondents ranking "classical music" 2nd, 3rd \& 4 th in Question 20 $(100 \%=261$ questionnaires $)$

\section{Analysis of the Results}

The questionnaire was formulated in such a way that distinctions between historical-stylistic, evaluative, and socioeconomic interpretations of the four main terms under consideration could be analyzed. Each of the nine descriptive phrases offered in question-type 6 (nos. 6, 10, 14, 18) can be classified into one of these three categories, and many of the twenty-four adjectives given in question-type 1 (nos. 1, 7, 11, 15) and question-type 2 (nos. 2, 8, 12, 16) either duplicate these phrases or offer near-synonyms. Because many of these adjectives are open to a variety of interpretations, they are not easily confined to one category. While the classification shown in Figure 2 is not a rigid one, it will be useful in understanding the loose framework under which the study was carried out, and for evaluating the statistical results. 
I. Historical-Stylistic

"a contemporary

phenomenon"

"a historical time

period"

"a musical style"

$\begin{array}{ll}\text { One category only } & \text { More than one category } \\ \text { ancient } & \text { conservative } \\ \text { baroque } & \text { innovative } \\ \text { romantic } & \text { radical } \\ & \text { traditional }\end{array}$

II. Evaluative

"an aesthetic

attitude"

"an established

norm"

"an ideal standard"

"a value judgement"

accessible
eccentric
enduring
entertaining
inaccessible
intellectual
progressive
serious

ethnic

familiar cheap

conservative

common

elite

expensive

innovative

radical

relevant

sophisticated

traditional

vulgar

III. Socio-Economic

"an ethnic

classification"

"a social standing"

cheap

common

elite

expensive

radical

relevant

sophisticated

vulgar

Figure 2

Descriptive phrases and adjectives used in the Questionnaire ${ }^{6}$ 
To encourage spontaneous replies, no attempt was made to be particularly systematic in the choice of these words and phrases. Naturally the alternatives offered reflect the biases and expectations of the investigators, but at the same time allow the respondents a wide variety of possible interpretations.

Before proceeding to a consideration of the four terms separately, it is important to emphasize the multiplicity of meanings elicited for each by the survey. In most cases respondents selected more than one answer for each question. Particularly noteworthy is the small number of single responses to question-type 6 .

\begin{tabular}{|c|c|c|}
\hline \multirow{7}{*}{$\begin{array}{l}\text { Question } 6-" \text { Classical" } \\
\text { musical style } \\
\text { time period } \\
\text { aesthetic } \\
\text { standard } \\
\text { established norm } \\
\text { value judgement }\end{array}$} & \multicolumn{2}{|c|}{ Single responses (\%) } \\
\hline & 8 & \\
\hline & 5 & \\
\hline & 2 & \\
\hline & 1 & \\
\hline & 0 & \\
\hline & .4 & Subtotal 16 \\
\hline \multicolumn{3}{|l|}{ Question 10 - "Popular" } \\
\hline musical style & 8 & \\
\hline social standing & 5 & \\
\hline ideal standard & .4 & \\
\hline contemporary & 17 & \\
\hline value judgement & 8 & Subtotal 38 \\
\hline \multicolumn{3}{|l|}{ Question 14 - "Folk" } \\
\hline musical style & 20 & \\
\hline ethnic classification & 15 & \\
\hline established norm & 2 & \\
\hline contemporary & .4 & \\
\hline value judgement & 1 & Subtotal 38 \\
\hline \multicolumn{3}{|l|}{ Question 18 - "Modern" } \\
\hline musical style & 2 & \\
\hline time period & 6 & \\
\hline ideal standard & .4 & \\
\hline aesthetic & 3 & \\
\hline contemporary & 8 & \\
\hline value judgement & 2 & Subtotal 21 \\
\hline
\end{tabular}

Table VII

Single responses to Questions 6, 10, 14, and 18 given in percentages $(100 \%=261$ questionnaires $)$ 
“CLASSICAL” (Questions 1-3; 6)

Most responses selected for the term "classical" may be organized into two large groups. "Historical-stylistic" (category I) characterizations are chosen most frequently. Thus the majority described "classical" as a "musical style" (85\%; single responses $8 \%$ ) and/or "a historical time period" (74\%; single responses $5 \%$ ), and considered it opposite in meaning to "romantic" $(40 \%)$ and "baroque" (35\%). The second major group of responses chosen was "evaluative" (category II). This is most clearly represented in responses to question 2, where "intellectual" (68\%), "enduring" (66\%), "accessible" (43\%), and "entertaining" (43\%) were most often selected as "applicable" adjectives. Furthermore, although it ranked third in question 6, "an aesthetic attitude" was nevertheless chosen by a significant proportion of the sample group ( $47 \%$, but only $2 \%$ single responses).

These results support the hypothesis that two different meanings of "classical" are at work:

(a) a narrow historical style period (category I);

(b) a broad classification of "serious art-music" based primarily on aesthetic (evaluative) criteria (category II).

The first meaning corresponds to the textbook definition of "classical music" as a term for the music of Western Europe composed between ca.1760 to ca.1820, and exemplified by the works of Haydn, Mozart, and Beethoven. Given the general bias of the sample group, one would expect this meaning to dominate the results. That this in fact occurs is demonstrated by the responses to question 3, where "Mozart" was not only chosen by almost all respondents $(98 \%)$, but was also unrivalled by any of the alternatives offered.

The second meaning emerges as a less well-defined concept. Despite the undeniable emphasis on evaluative criteria shown by the replies to question 2, there was a noticeable reluctance to admit "an aesthetic attitude" as an appropriate description in question $6 .^{7}$ Furthermore, the significant but disparate choices of composers other than "Mozart" in question 3 discloses a general lack of agreement about the extents and limits of this wider meaning.

The main terminological problem which arises here is the existence of what may suitably be called a "continually oscillating term." In other words, the connotation of one of the above meanings would seem to impinge on the other, regardless of the user's conscious intention. 
Thus, on the one hand, the wider meaning of the term "classical music" is limited by the historical-stylistic criteria of the narrower meaning. Responses to question 3 clearly reveal this influence. Interestingly, the ranking of composers after "Mozart" (i.e., Chopin, Chaikovsky, Bartók, Palestrina, Machaut, Willan, Shankar) is a sequence showing progressively greater distance from Mozart, both chronologically and stylistically. On the other hand, attempts by specialists notwithstanding, the use of the term in its narrower meaning continues to carry with it the connotation of "high aesthetic worth." This demonstrates that the best-formulated "textbook" definitions do not always succeed in driving out unwanted connotations.

\section{“POPULAR” (Questions 7-10)}

The majority of the responses chosen were "evaluative" (category II) or "socio-economic" (category III) descriptions. "Popular music" was felt to be "entertaining" (78\%), "common" $(70 \%)$ and "relevant" $(39 \%)$, and was considered opposite in meaning to "elite" (51\%), "inaccessible" (47\%) and "serious" $(41 \%)$. The characterizations "a value judgement" (36\%; single responses $8 \%$ ) and "a social standing" (33\%; single responses $5 \%$ ) were chosen frequently in question 10 , although they ranked third and fourth respectively. The two most frequently chosen responses to question 10 were "a contemporary phenomenon" (61\%; single responses a remarkable $17 \%$ ) and "a musical style" (45\%; single responses $8 \%$ ), both of which are "historicalstylistic" characterizations (category I).

The interpretation of "popular music" which emerges from this is: "a contemporary style of music with mass entertainment as its primary function (categories I, II, and III)." This neatly combines elements from all of our main categories, but problems emerge when the results are examined more carefully. Especially troublesome is the fact that although "a musical style" was frequently chosen as an apt description in question 10, apparently divergent styles are represented by the preferred choices in question 9 (Clark, 74\%; B.T.O., 67\%; Baez, 60\%; Boston Pops, 59\%; West-Side Story, 47\%; Armstrong, 43\%). This anomaly can be resolved by making one of the following assumptions: ${ }^{8}$

(a) "musical style" is not a well formulated or generally understood concept;

(b) respondents found more stylistic similarity among the items singled out in question 9 than disparity; 
(c) musical style per se was not an important criterion for responses chosen.

The third assumption suggests that, in addition to the more particular interpretation of "popular music" given above, two more general meanings were apparently influential:

(1) any music whose chief function is entertainment (categories II and III);

(2) any music "of the people" (category III).

Neither of these very general meanings is limited to a particular musical style, and thus their application could account for the range of responses to question 9 . If, on the other hand, either of assumptions (a) or (b) is true, it would seem that style and other criteria are so inextricably bound up together that they cannot be separated. While "classical music" oscillates between the more closely related categories I and II, "popular music" partakes of all three without belonging clearly to any.

"FOLK" (Questions 11-14)

Among the responses most frequently selected, one can distinguish between "stylistic" (category I) descriptions as opposed to "socio-economic" (category III) and "evaluative" (category II) ones. On the one hand, most respondents chose "a musical style" ( $70 \%$; single responses, a high $20 \%$ ) as the most suitable characterization, and furthermore considered it to be "traditional" $(79 \%) .{ }^{9}$ On the other hand, it was frequently described as "an ethnic classification" (64\%; single responses, a significant $15 \%)$. It was considered to be "ethnic" $(82 \%)$, "familiar" (67\%), "accessible" (59\%) and "relevant" (42\%), and was judged to be opposite in meaning to "sophisticated" (57\%) and "elite" (54\%).

These results suggest that two quite different meanings of "folk music" are used by the respondents:

(1) any "ethnic" music (category III);

(2) a particular commercial stylization of one kind of traditional Anglo-American music (categories I and II). In a literal sense, the second meaning is a particular subset of the first. However, since one is unlikely to view one's own culture as "ethnic," the two meanings can be considered mutually exclusive in the context of this study. Furthermore, the "musical style" identified in (2) becomes in a sense divorced from the ethnic group which originated it, and therefore ceases to be "folk music" in the sense of (1). 
An analysis of the responses indicates that, although most of the survey group accorded considerable weight to the first interpretation of "folk music," this was often little more than an acknowledgement of the existence of this general meaning. The dominance of the second, more specific meaning is clearly shown in question 13 , where it was obviously used as the determining factor in selecting exemplary performers, composers, and music. "Lightfoot" (76\%), "Baez" (75\%), and Dylan (73\%) were chosen far more often than the other alternatives. By contrast, examples of ethnic music in the general sense ("Bartók," "Shankar"), including diverse manifestations of the Afro-American tradition ("blues," "Porgy and Bess") were relatively ignored.

Again, a terminological problem arises from the simultaneous use of two distinct meanings for a single term. Although most respondents were able to restrict themselves to one meaning in question 13, the two meanings were confused in all of the other questions. It is interesting that, in a subtle way, stylistic elements of the second meaning can become associated with the general socio-economic element of the first meaning. This may be the origin of the common misconception that "ethnic music" in general is never "sophisticated" or highly artistic ("elite").

\section{“MODERN" (Questions 15-18)}

Almost all of the favored responses in this section of the survey were "historical-stylistic" ones (category I). "Modern music" was most often described as "a musical style" (54\%; single responses $2 \%$ ), "a historical time period" (45\%; single responses $8 \%$ ). In addition, it was considered to be opposite in meaning to "ancient" $(84 \%)$ and "romantic" $(40 \%)$. The main evaluative choices (category II) were the adjectives "progressive" (74\%) and "radical" (55\%).

Again, the overall results support the hypothesis that respondents were using two quite different and, in this case, mutually exclusive meanings of the term "modern music":

(1) a contemporary "art music" style (category I);

(2) the style of a more "popular" commercial kind of contemporary music (category I).

This hypothesis would allow us to explain some apparent anomalies in the responses. The first meaning would explain the frequent selection of "Stockhausen" $(65 \%)$ and "Bartók" 
(48\%) in question 17; the second would account for the high ranking of "Jesus Christ, Superstar" $(46 \%)$ in the same question. The existence of these disparate meanings would also explain the contradictory answers in question 16, where "modern" was seen as both "accessible" $(28 \%)$ and "inaccessible" $(15 \%) .{ }^{10}$ In one questionnaire, an explicit example of the second meaning was given by a respondent from HMU 120 who bracketed "modern" and "popular" in question 20 with the added remark "interchangeable."

The equation of the term "modern music" with some kind of popular, commercial type may be evidence of the alienation felt by many respondents toward contemporary "art-music." In addition, we note that "classical" is chosen as an appropriate opposite for "modern" by $59 \%$, just as "modern" was chosen by $58 \%$ as the opposite of "classical" in question 1. Although this is most obviously a comparison of historical periods, the "evaluative" meaning of "classical" is probably influential, whether intentionally or not. The small percentage of those who chose "classical" as applicable on question $16(11 \%)$ suggests that the two are incompatible regardless of which meanings are intended.

The results of this survey highlight the problematic nature of our terminology for classifying music. It is not simply the fact that some terms have several different meanings. The property of polysemy is characteristic of many words, and is not in itself sufficient to render the use of a given term troublesome, provided the context makes the intention clear. Nor is the difficulty limited to the fact that the boundaries ("terminals") of a particular meaning may be unclear. On the contrary, one may reasonably concede that this property is shared by most concepts in everyday language. ${ }^{11}$

Instead, this study has demonstrated how several types of terminological problems produce adverse effects in the specialized field of music. Each of the four terms studied is frequently overburdened with the task of representing, explicitly or implicitly, different kinds of classifications based on elements of historical origin, musical style, evaluative judgement, aesthetic value, function, and social standing. The resulting terminological problems cause and reinforce many widely held misconceptions about various kinds of music. The authors can offer no remedy, but these pages should serve to alert both 
professional and amateur users of this classification system of its many pitfalls.

This study has made a case for one kind of interpretation of the data presented in the tables. It must be emphasized in closing that other kinds of interpretations could be derived from the same data. A different set of categories (see Figure 2, I-III), for instance, might produce a different result (e.g., positive vs. negative adjectives and descriptive phrases; extrinsic vs. intrinsic qualities, etc.). Also, the various sub-groups which made up the sample could have been compared in more detail. What could be learned, for instance, from a detailed comparison of the most advanced group of students (HMU 454, HMU 354, HMU 357) with the least advanced (MUS 100)? How deep do the differences between first year (HMU 120) and second year students (HMU 220) go? What about the group studying nonWestern, or popular music (MUS 200, HMU 354), compared to the larger group? It has not been possible to pursue all of these subjects in this brief report, but it is hoped that the main objectives of the survey have been fairly and fully set forth. 


\section{NOTES}

1. We wish to emphasize that this informal scheme is only the most recent manifestation of the West's preoccupation with the classification of music, from the classic Boethian system through the various divisions proposed by seventeenth- and eighteenth-century writers based on stylistic, functional, and "class" criteria.

2. In spite of this, one respondent found the questionnaire "extremely poorly thought out ... ; these terms can mean many things to many people... I know I certainly use the terms in many various ways - not only one way as our answers [ sic ] to this survey would suggest."

3. One of the latter group studied our questionnaire with great care for perhaps ten minutes before declaring himself unable to answer it. The higher degree of sophistication which he brought to the task made him unable to respond with the desired spontaneity.

4. This is known as the "ordinal or position bias." See Warwick and Lininger 1975:147.

5. The tendency of all such surveys to yield a high degree of similarity between the respondent and his/her conception of social norms may be at work in this case. This kind of response is called "acquiescence" or "social desirability" in Warwick and Lininger, 1975:145-46.

6. The adjectives "classical," "popular," and "modern" have been omitted.

7. Analysis of the "aesthetic" aspect is further complicated by the fact that meaning (1) is a subset of meaning (2).

8. The possibility that the respondents simply disagreed among themselves is ruled out by the high percentage of responses in all questionnaires to six of the alternatives offered in question 9.

9. "Traditional" was the only adjective from category I offered for choice in questions 11 and 12 . The same is true in the parallel questions under "popular."

10. While these figures do not seem close enough, or high enough, to be significant, the comparable figures for this pairing under "classical" (question 2) were 43\% - 5\%; "popular" (question 7) 9\% - 47\%; and "folk" (question 12) 59\% - 2\%. The question of accessibility versus inaccessibility was resolved much more easily and decisively in the case of the first three terms studied.

11. See Lakoff 1975:221-71.

"Logicians have ... engaged in the convenient fiction that sentences of natural languages ... are either true or false ... or have a third value often interpreted as 'nonsense'.... Yet students of language ... have long been attuned to the fact that natural language concepts have vague boundaries ... and that, consequently, natural language sentences will very often be neither true, nor false, nor nonsensical, but rather true to a certain extent and false to a certain extent ...." (221)

\section{REFERENCES}

\section{LAKOFF, G}

1975: "Hedges: A Study in Meaning and the Logic of Fuzzy Concepts," in Hockney, D.J., Harper, W., and Freed, B., eds., Contemporary Research in Philosophical Logic and Linguistic Semantics. Boston: D. Reidel.

WARWICK, D.P., and LININGER, C.A.

1975: The Sample Survey: Theory and Practice. New York: McGraw-Hill. 